\title{
Plasmonic Sensor based on Microstructure PCF: Performance Analysis with Outside Detecting Approach
}

\author{
Md. Biplob Hossain ${ }^{\mathrm{a}, 1, *}$, Md. Nazmus Sakib ${ }^{\mathrm{b}, 1}$, and Md. Sanwar Hossain ${ }^{\mathrm{c}}$
}

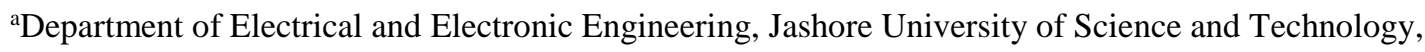 \\ Jashore-7408, Bangladesh \\ ${ }^{b}$ Department Electronics \& Telecommunication Engineering, Rajshahi University of Engineering \& Technology, Rajshahi 6204, \\ Bangladesh \\ 'Department of Electrical and Electronic Engineering, Bangladesh University of Business and Technology, Dhaka-1216, \\ Bangladesh
}

\section{(biplobh.eee10@gmail.com, sakib.ruet.ete@gmail.com, sanwareee@gmail.com)}

\footnotetext{
${ }^{1}$ These authors contributed equally to this work and co-first authors.

${ }^{*}$ Corresponding author at: biplobh.eee10@gmail.com (Md. Biplob Hossain)
}

Abstract - In this microarticle, we design a microstructure photonic crystal fiber (PCF) based external sensing surface plasmon resonance (SPR) sensor. The performance of the design is numerically evaluated incorporating the finite element method (FEM) with Perfectly Matched Layer (PML) boundary condition of scattering case. Modal analysis is performed using finer mesh anlaysis. At the optimized thickness (40nm) of chemically stable gold(Au) layer, the ever been maximum reported wavelength sensitivity (WS) and standard amplitude sensitivity (AS) are to 75,000 nm per RIU and 480 per RIU correspondingly. The sensor also exposed high polynomial fit $\left(R^{2}=0.99\right)$ as well as high figure of merit (FoM) of 280.77 per RIU. Since very much high sensitivity, high detecting range and figure of merit, lowing the cost of fabrication, the proposed design can be a pleasant competitor in detection of the analyte refractive index (RI). At the last, to prove performance ability of our designed sensor all the performance parameter calculated results compare with the existing sensors.

Keywords— FOM; SPR; PCF; Microstructure; PML;

\section{INTRODUCTION}

The SPR associating PCF sensor works on the theme of interaction between the incident light inside the PCF and the metal thin layer surface [1-2]. In the advancing world of science and technology, the PCF based SPR sensor is widely appreciated for its appliance in biomolecular sensing and detecting [3-8], temperature sensing [3], pollution sensing [4], environmental sensing and detecting [4], water testing[4], antigen-antibody interaction [5], medical diagnosis [6] etc. The PCF fiber is one kind of circular glass fiber with air holes inside it [7]. These air holes act as lower density medium that causes total internal reflection inside the fiber. When light beams pass through the core of the fiber they create evanescent field of $\mathrm{x}$ polarized and y polarized light that penetrates to the layer of the metalic thin film $[4,7,8]$. During the time of hitting the polarized light of pole to the metal film, they release free electron from the surface of the 
metal and creates surface plasmon wave [8]. In case of a particular wavelength, the plasmon electrons of the surface match with the incident light beam frequency of that corresponging wavelength and this causes a huge energy transfer from the light beam to free electron of metal [9]. At that point we get a sharp loss peak in the resonance curve. This wavelength is called resonance wavelength [9]. The unknown analyte can be detected and sensed observing this loss peak. The core background is filled up by fused silica for its low temperature sensitivity. The plasmonic material is another issue for sensing performance [10]. Several metals like gold, silver, titanium di-oxide ( $\mathrm{TiO} 2)$, aluminium etc are used as plasmonic material [7]. Although silver shows very high resonance peak, it exhibits chemical unstability due to its oxidization which can be prevented using graphene layer [11]. But this additional layer causes extra fabrication cost. So for the chemical stability and low fabrication cost, gold is mostly preferred plasmonic material $[7,8,10]$.

Several SPR sensors were introduced previously with different outcome in the literature. In recent, S. Chakma et. al. in literature [7] recommended a gold coated PCF based SPR design which spectacles maximum WS of $15,000 \mathrm{~nm}$ per RIU with maximum AS of 230 per RIU. A modified D-shaped design was offered very recently by L. Nannan in literature [12] which shows comparatively less sensitivity of 2900 nm per RIU than the others. An Ag coated hollow-core PCF sensor was reported by M.B. Hossain, et. al in literature [13]. This sensor reduced fabrication difficulties but shows less WS of $21000 \mathrm{~nm}$ per RIU than the model offered in this microarticle.

Our proposed design exhibits a very high WS of 75,000 nm per RIU and standard AS of 480 per RIU. The sensor exhibits high sensing range of 1.33 RIU to 1.41 RIU. The performance is evaluated numerically by combing the finite element method/FEM with Perfectly Matched Layer (PML) following the boundary condition of scattering case. Modal analysis is performed using finer mesh analysis. For very high sensitivity, high detection range, high linearity and FoM, less fabrication cost, the proposed sensor can be a great candidate in the recent research activities. 


\section{Arrangement of Structural Design}

The schematic cross sectional outlook of the PCF microstructure SPR design which is proposed in this microarticle is sketched in Figure. 1(a).

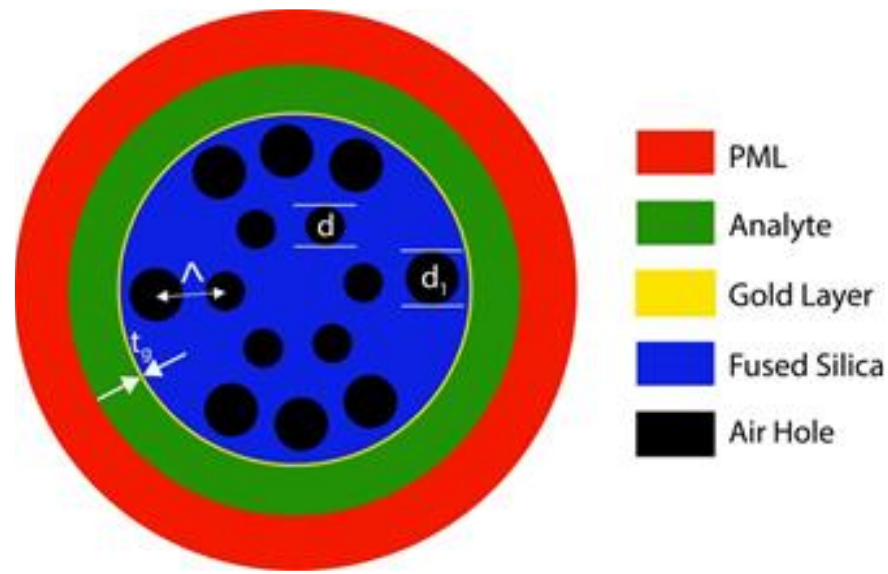

(a)

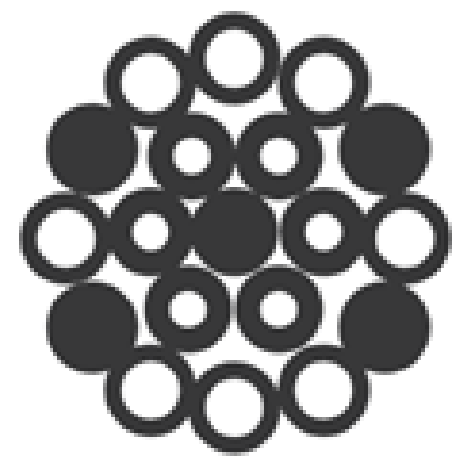

(b)

Figure. 1 (a) The 2-Dimensional and (b) Stack view of the offered design.

In the hybrid structure, the first ring is a hexagonal lattice and the second ring is a circular lattice with four missing air holes. These missing air holes are deployed to make strong evanescent field. In Figure.1(a) defines the core mode with $\mathrm{x}$-polarization where the electromagnetic wave propagates through $\mathrm{x}$ - axis. In Figure.1 (b) shows the Stack view of the sensor to be proposed. In phase identical point, the power is transmitted from foundamental core mode to foundamental SPP mode. As we proposed an asymmetric structure a strong birefringence will occur through the detection process. The centre to centre distance between two adjacent air hole is called pitch $(\Lambda)$. The dia of the first ring air hole is denoted by ds which is 0.57 of pitch. Again the diameter of the air hole of second ring is defined as 0.78 of pitch. The main background material is fused silica [9].The RI of the silica material calculated by the Sellmier equation which is denoted by $[13,14]$ :

$$
n^{2}(\lambda)=1+\frac{B_{1} \lambda^{2}}{\lambda^{2}-C_{1}}+\frac{B_{2} \lambda^{2}}{\lambda^{2}-C_{2}}+\frac{B_{3} \lambda^{2}}{\lambda^{2}-C_{3}}
$$

Here, $\mathrm{n}$ is the effective RI of the fused silica function of wavelength $(\lambda)$ which is measured in $\mu \mathrm{m}$ scale.Where $B_{1}, B_{2}, B_{3}, C_{1}, C_{2}$ and $C_{3}$ are the Sellmier equation constant those are taken from Ref. [14]. As $\operatorname{gold}(\mathrm{Au})$ is a chemically stable material used for external sensing layer which thickness $(\operatorname{tg})$ is variable and 
developed thin film by chemical vapor deposition(CVD) technique [14].The dielectric function of Au is received from Drude-Lorentz model $[15,16]$.

$$
\epsilon_{A u}=\epsilon_{\infty}-\frac{\omega_{D}^{2}}{\omega\left(\omega+j \gamma_{D}\right)}-\frac{\Delta \in \Omega_{L}^{2}}{\left(\omega^{2}-\Omega_{L}^{2}\right)+j \Gamma_{L} \omega}
$$

Where, $\varepsilon A u$ is the permittivity of $\mathrm{Au}$ and $\varepsilon_{\infty}=5.9673$ is the permittivity of Au at high frequency. The assessment of other coefficients are received from literature [16]. The Au layer is positioned the outer surface to identify the sample deligently. The optimum thickness of the gold layer is 40nm [15].Then the liquid sample passing layer as analyte layer which is $1.5[\mu \mathrm{m}]$ thickness. To diminish the reflection of light, the next layer is placed with PML, which is $1.5[\mu \mathrm{m}](10 \%$ of the PCF diameter) and we also applied the sensing scattering boundary condition to imbibe the incoming evanescent field wave radiate from the PCF. FEM based mode solver COMSOL Multiphysics is used to design the sensor and also analyze the different modes. The mathematical calculation is formulated by MATLAB. We used finer mesh for perfect calculation.

\section{SIMULATION OUTCOMES AND PERFORMANCE ANALYSIS}

The performance of the offered sensor is investigated by considering total number of elements 20,256, number of vertex elements 128 , number of boundary elements 1,628 and the quality of the element is 0.7988. To observe the sensitivity of a sensor, we need to obtain the confinement loss curves with respect to different parameters. The loss of the confinement curve is achieved using the following formula:

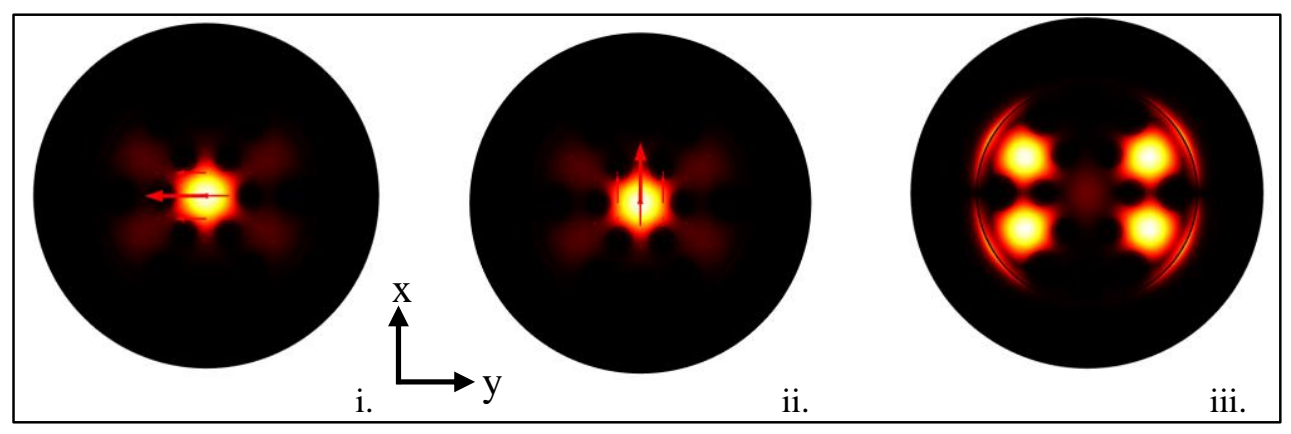

(a) 


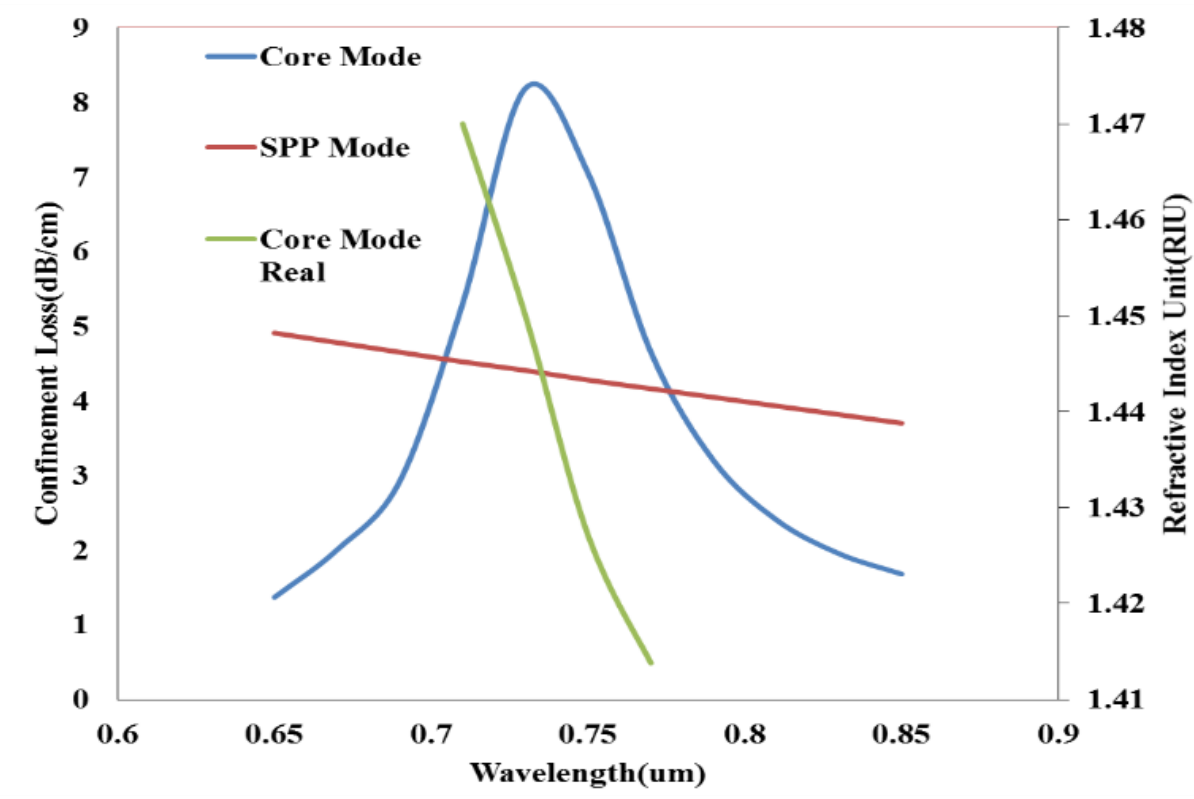

(b)

Figure.2: (a) The EM field distribution of fundamental mode of the (i) x-polarized core, (ii) y-polarized core, (iii) y-polarized SPP and, (b) matching relation of dispersion phase between the mode of the fundamental core and SPP at $\mathrm{n}_{\mathrm{a}}=1.445$ and $\mathrm{t}_{\mathrm{g}}=40 \mathrm{~nm}$.

$$
\alpha(d B / c m)=8.68 \times k_{0} \operatorname{Im}\left(n_{e f f}\right) \times 10^{4}
$$

Here, mode of the core of imaginary refractive index is expressed by " $\operatorname{Im}\left(\mathrm{n}_{\mathrm{eff}}\right)$ ", the number of waves is indicated by "ko", where the operating wavelength is expressed by $\lambda$ as it is referred in [16]. The curve of confinement loss is appeared in Figure.2(a) with respect to the wavelength for analyzing the variation of $\mathrm{RI}$ in between 1.41 and 1.48 .

In the case of a specific analyte, the directed mode of the fundamental core and SPP is coordinated at 1.445 RIU as demonstrated in Figure. 2(b). It is observed from the figure. 2(b), it shows that the real core mode portion and the SPP mode are intersected at the point of wavelength $0.74 \mu \mathrm{m}$. The wavelength at which the modes of two cases are intersected is called phase matching wavelength and a sharpe resonance peak is happened at that corresponding wavelength. In case of the resonance wavelength, the maximum energy is transferred to the plasmonic mode from the from the core mode. The Figure. 2(a) depicts the xpolarizd core mode of EM field, y-polarised core mode and the SPP mode for the case of wavelength at which the phases are matched. To test sensor output, confinement loss is one of the keys to enhance the sensor performance which is calculated employing the equation (3). As it is seen from the Figure.3(a), the variation the resonance peak loss is shifted to different wavelength due to different analyte profile. With increasing the analyte RI from 1.33-1.41, the loss depth also incraesing drastically.So that as the analyte RI 
increases, the phase coordinating point shifted to greater wavelength. After obtaining loss curves, we calculated the sensitivity of the sensor to evaluate its performance. Therefore, it can be made a conclusion that the deviation of the analyte profile of RI is one of the significant ways in analyzing the output of the offered design which is measured by:

$$
S_{\lambda}(n m / R I U)=\Delta \lambda_{p_{e a k}} / \Delta n
$$

Here, $\Delta \lambda$ peak indicates the wavelength peak variation, the variance of the analyte RI is symboled by $\Delta \mathrm{n}$. By observing the shifting the peak of the resonance curves, we can achieve the sensitivity of performance in terms of wavelength by employing the interrogation method of wavelength. It has been calculated that the maximum WS is $75,000 \mathrm{~nm}$ per RIU.

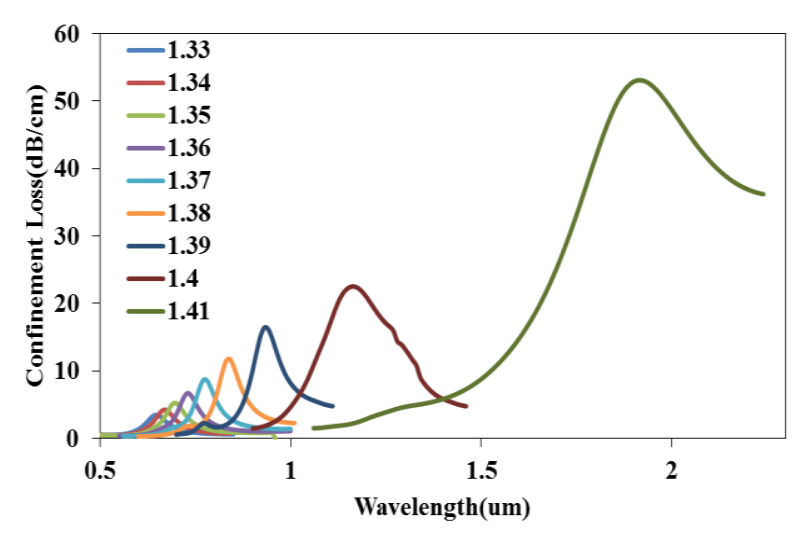

(a)

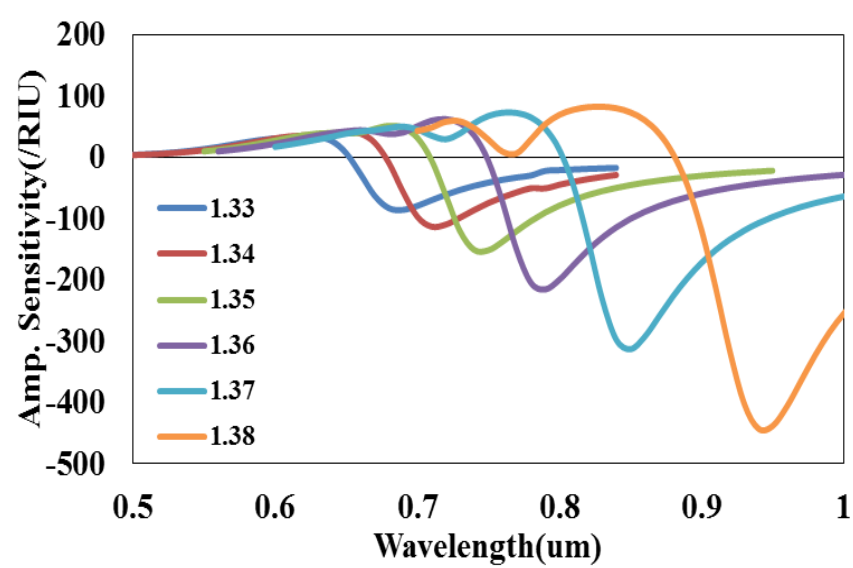

(b)

Figure. 3(a) Variation of loss profile of analyte RI from $1.33 \mathrm{RIU}$ to $1.41 \mathrm{RIU}$, (b) AS with $\Lambda=2 \mu \mathrm{m}, d \mathrm{~s}=0.57 \Lambda, d \mathrm{l}=0.78 \Lambda$, and $t g=40 \mathrm{~nm}$.

Another technique is the amplitude interrogation procedure to measure the sensor sensitivity. In this approach, the sensitivity of sensor in case of measuring the amplitude is given by:

$$
S_{A}\left(R I U^{-1}\right)=\frac{1}{\alpha(\lambda, \eta)} \times \frac{\partial(\lambda, \eta)}{\partial \eta}
$$

Here, $\alpha(\lambda, n)$ reveals the loss of propagation and $\alpha(\lambda, n)$ indicates the loss gap. As it is exhibited from Figure.3(b), the maximjum AS is reported to 480 per RIU in terms of wavelength and is plotted for analyte RI profile ranging between 1.33 to 1.38 RIU. The signal to noise ratio or SNR is another major factor to be investigted the superiority of the sensor which can be found with the Figure of Merit (FOM). The FOM is got commencing the following equation: 


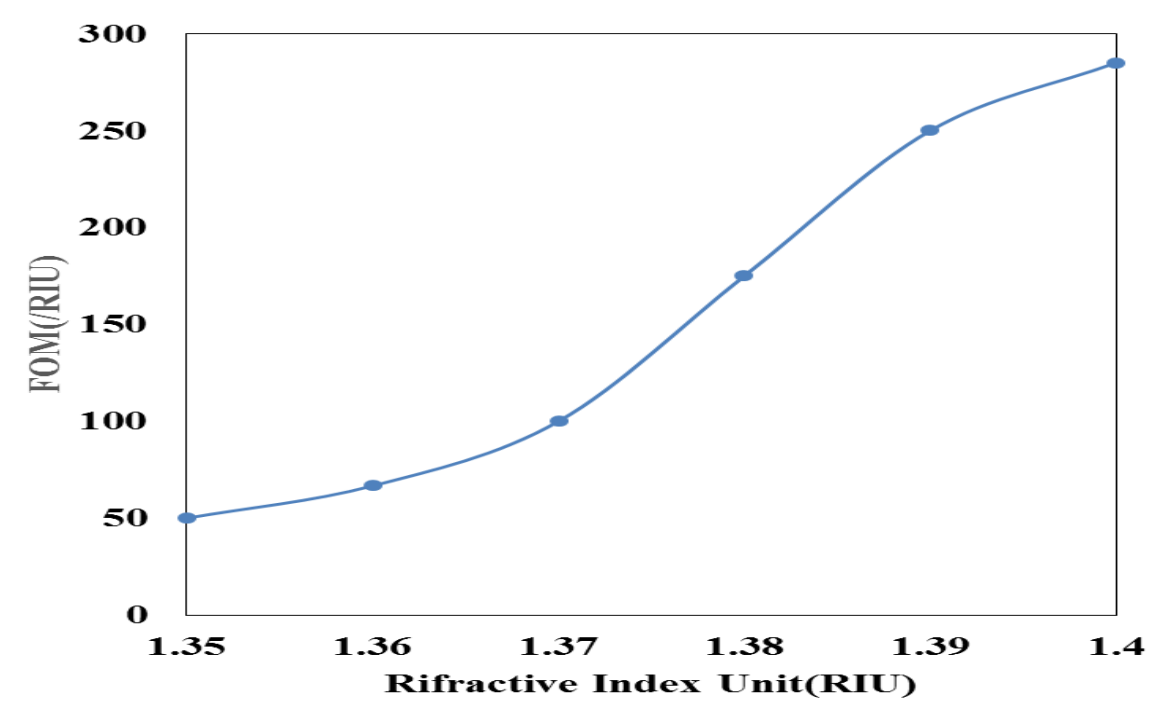

Figure. 4: FOM characteristics representation.

Here, the FWHM means "full width at half maximum". The improved SNR results in a higher spectrum of detection of the design. From the equation (6), we observe that higher FOM can be obtained by increasing sensitivity and decreasing FWHM.In Figure.4, we take FOM with respect to RI(from 1.35 to 1.40) and it is visible that with the increasing analyte refractive index (na) the sensitivity is increasing but the and the FWHM reduce as the narrow resonance peak is observed.Therefore,the maximum FOM is found $280.77 \mathrm{RIU}^{-1}$ at $\mathrm{RI} \mathrm{na}=1.40$. The predictor of a good sensor is a high linearity characteristic. The linearity fitting of this sensor is Illustrated in Figure.5 with analyte RI variation ranging 1.32 to 1.42 RIU.

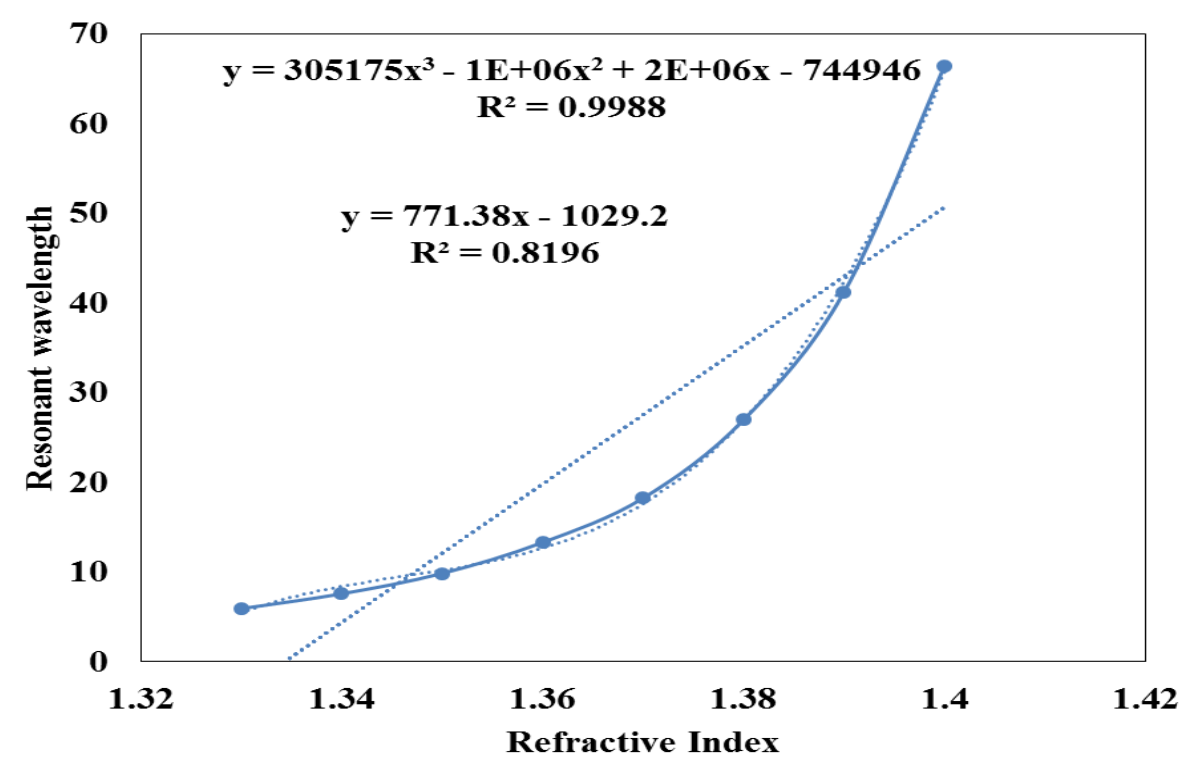

Figure. 5: Demonstration of the sensor's line fitting characteristics with varying RI from 1.32 to 1.42 RIU. 
From Figure.5, we can see that our offered design shows high linearity fitting with $\mathrm{R}$ square value of 0.8196 and high polynomial fitting with R square value of 0.9988 . Here, y indicates the wavelength of the resonance and $\mathrm{x}$ denotes the analyte's RI.

Table 1:Performance Comparison with the previously designed existing sensors.

\begin{tabular}{|c|c|c|c|c|c|}
\hline $\begin{array}{c}\text { Ref. } \\
\text { No. }\end{array}$ & $\begin{array}{c}\text { WS } \\
(\mathrm{nm} / \\
\text { RIU })\end{array}$ & $\begin{array}{c}\text { AS } \\
\left(\mathrm{RIU}^{-1}\right)\end{array}$ & $\begin{array}{c}\text { FoM } \\
(\mathrm{RIU})\end{array}$ & $\begin{array}{c}\text { Polynomial fit } \\
\left(R^{2}\right)\end{array}$ & $\begin{array}{c}\text { Detecting } \\
\text { Range (RIU) }\end{array}$ \\
\hline$[2]$ & 20000 & 1054 & N/A & N/A & $1.18-1.36$. \\
\hline$[7]$ & 15,000 & 230 & 45 & N/A & $1.42-1.46$ \\
\hline$[8]$ & 8000 & 230 & 45 & 0.9702 & $1.42-1.46$ \\
\hline$[9]$ & 9,800 & 1,086 & N/A & N/A & $1.33-1.43$ \\
\hline$[13]$ & 21000 & 2456 & N/A & 0.7855 & $1.33-1.42$ \\
\hline$[14]$ & 8000 & 700 & 138 & 0.7971 & $1.20-1.40$ \\
\hline$[15]$ & 5000 & 396 & 47 & 0.9788 & $1.33-1.39$ \\
\hline$[16]$ & 4000 & 478 & N/A & N/A & $1.33-1.37$ \\
\hline Proposed Sensor & 75,000 & 480 & 280.77 & 0.9988 & $1.33-1.41$ \\
\hline
\end{tabular}

To prove performance ability of our designed sensor all the performance parameter calculated results compare with the existing sensor put on the table 1. From the table. 1, it is evident that our designed sensor displays better results with higher sensitivity, more sensing capacity and also the number of more detection range with compare the existing sensor.

\section{V.CONCLUSIONS}

In this microarticle, a highly wavelength sensitive microstructure PCF based SPR design is proposed, where the stable material gold external sensing method is used as a plasmonic material. The sensing output is analyzed using the FEM-based approach and the maximum AS is 480 per RIU along with the maximum WS $75000 \mathrm{~nm}$ per RIU at the detection range in between 1.33 to $1.41 \mathrm{RIU}$. As the sensor is highly sensitive, the maximum FOM is found that 278.77 per RIU. All the performance results are obtained by the simulation in the COMSOL Multiphysics software where the numerical analysis is performed by employing PML for the boundary condition of scattering case. In this analysis, the mesh size was kept as small as possible.

\section{REFERENCES}

[1]Rifat, Ahmmed A., et al. "Photonic crystal fiber based plasmonic sensors." Sensors and Actuators B: Chemical 243 (2017): 311-325.

[2] Haque, Emranul, et al. "Surface Plasmon Resonance Sensor Based on Modified D-Shaped Photonic Crystal Fiber for Wider Range of Refractive Index Detection." IEEE Sensors Journal 18.20 (2018): 8287-8293. 
[3] Mollah, M.A., Islam, S.R., Yousufali, M., Abdulrazak, L.F., Hossain, M.B. and Amiri, I.S., 2020. Plasmonic temperature sensor using D-shaped photonic crystal fiber. Results in Physics, 16, p.102966.

[4]Y. Lu, X. Yang, M. Wang, and J. Yao, "Surface plasmon resonance sensor based on hollow-core PCFs filled with silver nanowires," Electron. Lett., vol. 51, pp. 1675-1677, 2015.

[5] Hossain, B., Kabir, A., Rahman, M., Roy, S., Abdulrazak, L.F., Hossain, S., Mondol, N., Rahman, M.H., Islam, K.Z. and Pathan, M.I., 2021. Hybrid structure based high performance SPR sensor: a numerical approach of structure optimization for DNA hybridization. Optical and Quantum Electronics, 53(1), pp.1-19.

[6] Hossain, M.B., Islam, M.M., Abdulrazak, L.F., Rana, M.M., Akib, T.B.A. and Hassan, M., 2020. Graphene-coated optical fiber SPR biosensor for BRCA1 and BRCA2 breast cancer biomarker detection: a numerical design-based analysis. Photonic Sensors, 10(1), pp.67-79.

[7] Hossain, M.B., Mahendiran, T.V., Abdulrazak, L.F., Mehedi, I.M., Hossain, M.A. and Rana, M.M., 2020. Numerical analysis of gold coating based quasi D-shape dual core PCF SPR sensor. Optical and Quantum Electronics, 52(10), pp.1-13. [8] Hossain, M.B., Hossain, M.S., Islam, S.R., Sakib, M.N., Islam, K.Z., Hossain, M.A., Hossain, M.S., Hosen, A.S. and Cho, G.H., 2020. Numerical development of high performance quasi D-shape PCF-SPR biosensor: An external sensing approach employing gold. Results in Physics, 18, p.103281.

[9] Rifat, A.A., Ahmed, R., Mahdiraji, G.A. and Adikan, F.M., 2017. Highly sensitive D-shaped photonic crystal fiber-based plasmonic biosensor in visible to near-IR. IEEE Sensors Journal, 17(9), pp.2776-2783.

[10] Sakib, M.N., Islam, S.R., Mahendiran, T.V., Abdulrazak, L.F., Islam, M.S., Mehedi, I.M., Kamrunnahar, Q.M., Momtaj, M., Hassan, M.W., Amiri, I.S. and Hossain, M.B., 2020. Numerical study of circularly slotted highly sensitive plasmonic biosensor: A novel approach. Results in Physics, 17, p.103130.

[11] Szunerits, S., Maalouli, N., Wijaya, E., Vilcot, J.P. and Boukherroub, R., 2013. Recent advances in the development of graphene-based surface plasmon resonance (SPR) interfaces. Analytical and bioanalytical chemistry, 405(5), pp.1435-1443.

[12] Luan, Nannan, et al. "Surface plasmon resonance sensor based on D-shaped microstructured optical fiber with hollow core." Optics express 23.7 (2015): 8576-8582.

[13] Hossain, Md Biplob, SM Riazul Islam, KM Tasrif Hossain, Lway Faisal Abdulrazak, Md Nazmus Sakib, and I. S. Amiri. "High sensitivity hollow core circular shaped PCF surface plasmonic biosensor employing silver coat: A numerical design and analysis with external sensing approach." Results in Physics 16 (2020): 102909.

[14] Sakib, Md Nazmus, et al. "High performance dual core D-shape PCF-SPR sensor modeling employing gold coat." Results in Physics 15 (2019): 102788.

[15]Akter, Sanjida, and SM Abdur Razzak. "Highly sensitive open-channels based plasmonic biosensor in visible to nearinfrared wavelength." Results in Physics 13 (2019): 102328.

[16] Rifat, A.A., Hasan, M.R., Ahmed, R. and Butt, H., 2017. Photonic crystal fiber-based plasmonic biosensor with external sensing approach. Journal of Nanophotonics, 12(1), p.012503. 